\title{
Experiências, Conhecimento Fenomenal e Materialismo
}

\author{
Wilson MendonçA \\ Universidade Federal do Rio de Janeiro \\ Julia Telles Menezes \\ Universidade Federal do Rio de Janeiro
}

\begin{abstract}
The claim that at least some of our mental states have qualitative, phenomenal features to which we have privileged cognitive access is intuitively plausible. Nevertheless, the claim is considered by many philosophers to be incompatible with a physicalist ontology. Some radical physicalists prefer simply to deny the existence of the qualitative character of our mental states, whereas other physicalists try to reinterpret the knowledge of the phenomenal character of our experience as the acquisition of an ability, i.e., as a sort of know-how in opposition to the acquisition of an information (know-that). The paper presents and examines critically some recent attempts to compatibilize the sui generis nature of phenomenal knowledge with the materialist claim that the content of this sort of knowledge is constituted entirely by physical facts.
\end{abstract}

Keywords: Qualia; phenomenal knowledge; phenomenal concepts; epistemic contextualism.

Muitos filósofos acreditam que pelo menos alguns dos nossos estados mentais (as chamadas "experiências" ou "estados fenomenais") têm aspectos ou caracteres qualitativos aos quais nós temos um acesso na perspectiva da primeira pessoa. $\mathrm{O}$ aspecto que pode ser discernido introspectivamente, o chamado "caráter subjetivo" de um estado fenomenal, é normalmente concebido como uma propriedade intrínseca a este estado, isto é, como uma qualidade ou propriedade que o estado mental tem independentemente das relações com outros estados e eventos (mentais ou não-mentais) que poderiam figurar na especificação de sua natureza funcional. Dito em uma terminologia filosófica que remonta a C. I. Lewis, foi mobilizada por Nelson Goodman e permanece em uso nos trabalhos de muitos filósofos contemporâneos: os qualia (plural de "quale") são não-funcionalizáveis.

Os aspectos qualitativos fenomenais são normalmente concebidos no quadro do realismo fenomenal. A noção de qualia é a noção de caracteres que são como são independentemente da "percepção" que deles possamos ter. Em outras palavras, os qualia constituiriam a matéria ou o objeto do conhecimento introspectivo: o subject matter a propósito do qual podemos formar, justificar e eventualmente verificar crenças fenomenais. Conhecimento requer plausivelmente crenças; e crenças requerem

Principia 15(3): 415-438 (2011).

Published by NEL — Epistemology and Logic Research Group, Federal University of Santa Catarina (UFSC), Brazil. 
plausivelmente conceitos. Portanto, a assunção natural que acompanha a tese da existência dos qualia diz que existem conceitos fenomenais especiais cuja aplicação permitiria a discriminação adequada de eventos mentais, mediante a identificação correta dos aspectos qualitativos que os distinguem fenomenalmente. Contudo, por mais especiais que sejam, os conceitos fenomenais são concebidos como instrumentos do conhecimento genuíno em uma perspectiva realista: sua aplicação pode, em casos favoráveis, revelar, mas não criar os qualia das experiências às quais eles se aplicam. Isto implica, entre outras coisas, que é pelo menos possível - e talvez seja real - uma vida mental feita de experiências que não são "introspectadas" pelo sujeito dessa vida mental, uma sequência de estados fenomenais qualitativos que não são apreendidos fenomenalmente pelo sujeito. Para isso, basta que o sujeito seja essencialmente incapaz de adquirir ou formar os conceitos fenomenais necessários. Talvez existam sujeitos desse tipo. Mas é razoável pensar que nós, seres humanos normais, temos vidas mentais mais complexas, que, além de estados fenomenais com aspectos intrínsecos próprios, nós temos também uma natureza reflexiva que nos permite descobrir introspectivamente os qualia das nossas experiências — ou pelo menos formar uma opinião considerada sobre os qualia das nossas experiências. O ponto relevante é que os conceitos fenomenais invocados na discussão filosófica sobre a mente consciente são vistos como representações mentais de "segunda ordem", uma ordem metafisicamente independente da "primeira ordem" onde estão situados os objetos desses conceitos (as propriedades identificadas por eles).

Os defensores da teoria dos qualia e dos conceitos fenomenais frequentemente apresentam suas considerações como a elaboração relativamente abstrata de um ponto de vista comum. Brian Loar, por exemplo, introduz assim sua teoria seminal sobre os estados fenomenais:

On a natural view of ourselves, we introspectively discriminate our own ex-
periences and thereby form conceptions of their qualities, both salient and
subtle. [...] What we apparently discern are ways experiences differ and
resemble each other with respect to what it is like to have them. Following
common usage, I will call these experiential resemblances phenomenal qual-
ities, and the conceptions we have of them, phenomenal concepts. Phenome-
nal concepts are formed "from one's own case". They are type-demonstratives
that derive their reference from a first-person perspective: 'that type of sen-
sation', 'that feature of visual experience'. (Loar 1997, p. 597)

Outros filósofos são de opinião que as relações introspectáveis de diferença e similaridade entre experiências determinam antes what it is like to be the subject of phenomenal states. Isto é, de acordo com a visão defendida por esses filósofos, as propriedades discernidas pela visão reflexiva fenomenal comum (os objetos dos conceitos fenomenais que nós formamos normalmente) são propriedades do sujeito das experiências, em oposição a propriedades das experiências, como na visão de Loar. 
Outra alternativa consiste na identificação das propriedades fenomenais introspectáveis com propriedades presentes na experiência, em oposição a propriedades da experiência. Os qualia são, em um sentido primário, propriedades dos objetos representados na experiência e, só secundariamente, propriedades atribuíveis às experiências ou aos sujeitos das experiências.

Esta forma de representacionismo a propósito do conhecimento fenomenal, defendida por alguns filósofos atuais, já tinha sido formulada por Goodman. Goodman parte da suposição de que existe uma relação entre a propriedade "objetiva" das coisas e a propriedade "representada" na experiência, isto é, a propriedade atribuível às coisas representadas. O exemplo preferido de Goodman é o das experiências visuais de cores. As cores estão, de acordo com Goodman, entre as propriedades objetivas das coisas. A atribuição de uma cor a um objeto envolve uma asserção sobre as cores aparentes do objeto em diferentes circunstâncias de representação visual. Em geral, Goodman parece pensar que é quase parte do conteúdo de uma atribuição de propriedades objetivas às coisas que as propriedades apresentadas pelas coisas nas experiências que delas fazemos em diferentes condições (atuais ou possíveis) obedecem a um padrão (pattern) mais ou menos especificado. Desacompanhada de boas razões para pensar que um determinado objeto teve alterada sua propriedade objetiva de ser azul, por exemplo, a afirmação de que o objeto agora parece verde deve ser interpretada como fazendo referência a uma propriedade presente na experiência (a propriedade do objeto representado na experiência), em oposição (i) à propriedade designada pelo predicado em "o objeto é azul", mas também (ii) às propriedades invocadas em "o objeto mudou de cor". Isto leva diretamente à visão representacionista dos estados fenomenais:

Roughly, then, to say that a thing looks green is to make a statement concerning a presented quality, a color quality of some presentation of the thing, while to say that a thing is green is to make a more complex statement concerning the color qualities exhibited by various presentations of the thing. Obviously, the color names are thus used in two different ways in ordinary language: in the one case for presented characters, which I shall hereafter call qualia; in the other, for properties of things. (Goodman 1951, p. 130)

Para os propósitos do presente trabalho, as diferenças entre os pontos de vista que atribuem propriedades fenomenais primariamente às experiências, aos sujeitos das experiências ou aos objetos representados na experiência são, pelo menos por enquanto, menos importantes que a assunção comum a eles. E esta afirma a existência de propriedades "internas" que podem ser discernidas na perspectiva da primeira pessoa mediante a aplicação de conceitos fenomenais. Isto deixa ainda em aberto a questão ontológica sobre os "objetos internos": os qualia são propriedades irredutivelmente não-físicas ou propriedades idênticas (ou talvez supervenientes) a propriedades físicas? A questão pode ser reformulada em termos epistêmicos: a informação 
adquirida mediante a aplicação correta de conceitos fenomenais às experiências é física ou não-física? Tipicamente, conhecimento é aquisição de informação física, apreensão de fatos diretamente físicos ou fatos funcionais definidos sobre uma base física. A questão é sobre se existe informação irredutivelmente não-física, apreensível pelo conhecimento fenomenal.

O que veio a ser denominado de "estratégia dos conceitos fenomenais" é um conjunto variado de argumentos engenhosos que pretendem fornecer respostas a estas questões. A estratégia dos conceitos fenomenais visa mostrar (i) que existe um hiato epistêmico (epistemic gap) entre o domínio dos processos físicos e o das experiências subjetivas, (ii) que as peculiaridades dos conceitos fenomenais explicam (tornam "justificada") a intuição negativa comum que emerge imediatamente da mera consideração da identidade de propriedades fenomenais e propriedades físicas, mas (iii) que o dualista comete um erro ao inferir um hiato ontológico do hiato epistêmico. De acordo com a estratégia dos conceitos fenomenais, nada impede que sejam idênticas as propriedades identificadas pelos conceitos físicos, por um lado, e as propriedades identificadas pelos conceitos fenomenais, por outro. Ademais, a diferença entre conceitos físicos e os conceitos fenomenais explicaria por que os referentes destes conceitos nos parecem radicalmente distintos.

\section{II}

A estratégia dos conceitos fenomenais é uma resposta, ou melhor: um conjunto de respostas, à versão mais atual do argumento que parte de premissas epistêmicas para chegar a uma conclusão metafísica sobre a verdade do dualismo — ou pelo menos sobre a falsidade do monismo materialista ou fisicista.

Para compreender a versão mais atual do argumento epistêmico pró-dualismo (o argumento do conhecimento) é necessário considerar, em primeiro lugar, uma peculiaridade do conhecimento. O conhecimento é um estado mental factivo. Isto quer dizer que a atribuição de conhecimento requer, como uma condição necessária, a verdade do seu conteúdo. De acordo com externalismo de conteúdo, outros estados mentais também exigem a existência de fatos externos aos estados mentais. Se o externalismo de conteúdo defendido por Hilary Putnam estiver certo, só é correto atribuir crenças sobre água àqueles sujeitos que vivem de fato em um mundo onde a microestrutura do líquido que sai das torneiras, corre nos rios, preenche os lagos etc. é $\mathrm{H}_{2} \mathrm{O}$. Nesse sentido, uma crença qualquer sobre água, por exemplo, também teria um componente factivo. Porém, o que distingue o conhecimento, como estado factivo, dos outros estados mentais é que o conhecimento é sempre e necessariamente a apreensão de fatos, isto é, de proposições verdadeiras. Conhecimento, mas não crença, por exemplo, implica a verdade. Se " $K p$ " representa o conhecimento de conteúdo $p$ e " $C p$ " representa a crença de conteúdo p, então (1) é um princípio 
válido da lógica epistêmica, ao passo que (2) não regula geralmente a atribuição de crenças.
(1) $K p \rightarrow p$
(2) $B p \rightarrow p$

$\mathrm{O}$ argumento do conhecimento está baseado em um Gedankenexperiment concebido por Jackson (1982). De acordo com a história contada por Jackson, Mary finalmente sai do quarto preto e branco e vê, pela primeira vez, sem a intermediação de um monitor preto e branco, um objeto vermelho, por exemplo, um tomate maduro. Mary já tinha um vasto conhecimento antes de passar pela nova experiência. De fato, de acordo com a história, ela já tinha o conhecimento físico completo dos fatos (fisiológicos, neurológicos, biológicos etc.) envolvidos nas experiências de cores. Para Jackson, contudo, deve-se assumir como bastante plausível a ideia de que Mary adquire um conhecimento novo no momento exato em que ela tem a primeira experiência visual de um tomate maduro. Este novo item de conhecimento é o que foi caracterizado na seção anterior como conhecimento dos aspectos qualitativos da experiência. É importante notar que a situação de Mary antes de sair do quarto e depois de sair do quarto é caracterizada em termos de conhecimento: Mary tinha todo o conhecimento físico e, ao sair do quarto, Mary adquire um novo conhecimento. $\mathrm{O}$ caráter factivo do conhecimento implica que as informações que Mary tinha antes de sair do quarto, bem como a informação que ela adquire ao sair do quarto, são verdadeiras. Mary já tinha apreendido todos os fatos físicos e agora apreende mais um fato. E fácil inferir daí que o novo fato apreendido é não-físico.

Isto depende, obviamente, da compreensão literal da premissa segundo a qual Mary tinha todo o conhecimento físico. Daniel Dennett talvez tenha sido o primeiro a observar que é muito difícil imaginar que alguém possa adquirir todo o conhecimento físico, mesmo de um âmbito restrito da física, como a física das experiências visuais das cores. E isto não porque é factualmente difícil adquirir tanto conhecimento, mas porque a noção de possuir todo o conhecimento físico talvez não faça sentido. Se ignorarmos agora esta dificuldade e considerarmos que não é absurda a descrição de Mary como uma pessoa que já apreendeu todos os fatos físicos, o argumento do conhecimento pode ser formalmente resumido do seguinte modo:

(3) Mary conhece todos os fatos que constituem o mundo físico.

(4) Mary adquire, ao sair do quarto, um novo conhecimento.

(5) Conhecimento implica a verdade.

$\therefore$ (6) Existem fatos não-físicos.

$\therefore$ (7) O fisicismo é falso.

Principia 15(3): 415-438 (2011). 


\section{III}

Defensores do materialismo responderam de diferentes maneiras ao argumento do conhecimento. A resposta mais radical veio dos chamados qualiaphobes, que negam peremptoriamente os aspectos qualitativos da experiência e, consequentemente, a noção de que eles poderiam ser objetos de conhecimento. Seja lá o que for que acontece com Mary quando ela sai do quarto, sua nova experiência não deve ser descrita como aquisição de informação ou apreensão de fatos. Menos radical é a resposta dos que concordam que Mary adquire um novo conhecimento, mas que interpretam o novo conhecimento como a aquisição de uma habilidade, de um knowhow. Se o novo conhecimento não é um caso de conhecimento proposicional (knowthat), então o argumento do conhecimento não leva à conclusão anti-materialista.

Em um sentido importante, as duas respostas materialistas esboçadas acima propõem a dissolução (ao invés da solução) do problema gerado pelo argumento do conhecimento. Elas correspondem ao que David Chalmers (2003) chama de "materialismo do tipo A". As considerações a seguir, por outro lado, giram em torno de algumas respostas materialistas sofisticadas dadas por "materialistas do tipo B", respostas que, (i) ao contrário da resposta dos qualiaphobes, aceitam a integridade dos aspectos qualitativos da experiência acessíveis à consciência fenomenal e (ii), ao contrário da resposta da hipótese da habilidade, interpretam a nova experiência de Mary como objeto genuíno de estado cognitivo de conteúdo informacional.

Para bloquear a conclusão anti-materialista do argumento do conhecimento, a resposta materialista sofisticada deve mostrar que a nova informação adquirida por Mary não implica o reconhecimento de fatos situados além do âmbito de fatos já apreendidos no quarto preto e branco. Os materialistas que adotam a estratégia dos conceitos fenomenais - praticamente todos os materialistas contemporâneos trabalhando sobre consciência (cf. Papineau 2006, p. 111) - procuram mostrar que os conceitos fenomenais são distintos dos conceitos físicos somente no nível conceitual e se referem às mesmas propriedades físicas denotadas pelos conceitos físicos nãofenomenais. Portanto, novo conhecimento não implica necessariamente novo fato. Em oposição a isso, a resposta materialista contextualista (representada por John Perry e Robert Stalnaker) reinterpreta a primeira premissa do argumento do conhecimento. Se o novo fato deve ainda ser um fato físico, os fatos anteriormente apreendidos por Mary não podem ser literalmente todos os fatos físicos, como estabelecia a primeira premissa do argumento do conhecimento. Do ponto de vista do materialista contextualista, Mary adquire, durante o confinamento, todo o conhecimento que pode ser adquirido por alguém na sua situação. O conteúdo informacional do novo conhecimento de Mary, por outro lado, é acessível somente a quem se encontra na nova situação de Mary. Isto pressupõe que existem informações de tal forma ligadas a um contexto que elas só podem ser adquiridas por quem se encontra nesse contexto. Se

Principia 15(3): 415-438 (2011). 
o novo conhecimento de Mary for desse tipo, nada impede que a nova informação seja física.

\section{IV}

A versão do materialismo contextualista defendida por Perry aproxima, em primeiro lugar, o novo conhecimento de Mary e os casos que Perry chama de "conhecimento recognicional" (recognitional knowledge). Estes são casos de localização no tempo e no espaço e também da identificação correta da pessoa que eu sou. Perry argumenta que o conteúdo do conhecimento recognicional não é adequadamente capturado pelo referencialismo semântico dominante. Este especifica o conteúdo do conhecimento como uma proposição que é verdadeira se e somente se as entidades designadas pelos termos que compõem a expressão linguística do conhecimento (os referentes dos termos subsentenciais) estão relacionadas entre si da forma representada pela expressão sentencial. Isto é o que Perry chama de "conteúdo oficial", "conteúdo referencial" ou "subject matter content" (Perry 2001a, 2001b). Este tipo de conteúdo não permite diferenciar os estados cognitivos, intuitivamente diferentes, expressos por (8) e (9):

(8) Eu estou no Rio de Janeiro. (Dito por por JTM, co-autora do presente trabalho.)

(9) JTM está no Rio de Janeiro.

(8) e (9) têm o mesmo conteúdo referencial, o mesmo subject matter content: a proposição que é verdadeira se e somente se JTM estiver no Rio de Janeiro. (O termo "Eu" (neste contexto) e o termo "JTM" são co-referenciais, isto é, designam a mesma entidade, a saber: Julia Telles Menezes.) Contudo, eu posso saber que estou no Rio de Janeiro, mas, por estar sofrendo de amnésia e não saber mais que eu sou Julia Telles Menezes, não saber que JTM está no Rio de Janeiro.

Para dar conta do papel desempenhado pelo estado mental expresso por (8) nas inferências teóricas e práticas relevantes - que são diferentes das inferências teóricas e práticas a partir de (9) — , nós precisamos, segundo Perry, de um novo tipo de conteúdo, o "conteúdo reflexivo". Este é a proposição cujas condições de verdade incluem, além das entidades às quais se refere o conhecimento, os próprios elementos do veículo representacional. Assim, o conteúdo reflexivo do conhecimento expresso por (8) é a proposição que é verdadeira se e somente se a pessoa designada pelo proferimento situado do termo "eu" por parte do proferidor de (8) estiver no Rio de Janeiro. É o fato de que parte da representação à qual está sendo atribuído conteúdo aparece na especificação do conteúdo que explica por que o conteúdo em questão é reflexivo.

Principia 15(3): 415-438 (2011). 
Casos análogos à situação de Mary podem ser construídos para ilustrar a tese de que, ao contrário do que diz o argumento do conhecimento, um novo conhecimento não implica a expansão da ontologia além dos fatos previamente apreendidos:

Gary has been trapped for a month in a windowless hut across from Little America, just off Interstate 80 in western Wyoming. (Little America is a gas station with a restaurant and souvenir shop. It has more gas pumps than any place in the world.) He has memorized an interstate road map. Garry knows all the facts about the locations of things along Interstate 80: the order of states, cities, towns, and villages as one progresses west to east along Interstate 80, from Berkeley through Reno, Salt Lake City, Little America, Cheyenne, Lincoln, and on through the mysterious East. But he isn't allowed to look out of his hut, so he doesn't know where he is. Eventually he escapes. He sees all the gas pumps, realizes he is in Little America, and immediately knows a number of facts that seem to be facts about where things are along Interstate 80 but that he didn't know before. (Perry 2001, p. 108)

Antes de ser libertado de seu quarto, Gary estava em condições de afirmar:

(10) Salt Lake City está a sudoeste de Little America.

É somente após a sua saída do quarto que Gary pode afirmar que:

(11) Este lugar é Little America.

A partir de (10) e (11) Gary pode inferir:

(12) Salt Lake City está a sudoeste deste lugar.

Na perspectiva da subject matter assumption os enunciados (10) e (12) possuiriam o mesmo conteúdo. Este é um problema bem conhecido das teorias da referência direta: o conteúdo referencial não dá conta da significatividade de enunciados que envolvem termos co-extensionais. A noção de conteúdo reflexivo, por outro lado, permite lidar adequadamente com o novo conhecimento recognicional expresso por (12). O conteúdo referencial de (10) e (12) é a proposição que é verdadeira se e somente se Salt Lake City estiver a sudoeste de Little America, enquanto que o conteúdo reflexivo de (12), mas não o de (10), é a proposição que é verdadeira se e somente se o objeto designado pelo proferimento situado do termo "deste lugar" for Little America.

Gary adquire, portanto, uma nova informação, uma informação que ele não tinha e não poderia ter enquanto estava confinado. Contudo, o novo fato apreendido por Gary é ainda um fato sobre a localização das cidades e dos postos de gasolina ao longo da estrada. O novo item de conhecimento é sobre o mesmo subject matter sobre o qual Gary já sabia tudo. Mas a aquisição da nova informação sobre sua

Principia 15(3): 415-438 (2011). 
localização na estrada tem consequências práticas e teóricas distintivas que podem ser explicadas mediante o recurso às alterações na dimensão reflexiva dos conteúdos dos estados mentais de Gary. Mutatis mutandis, isto se aplica também ao caso de Mary. A distinção entre conteúdo referencial e conteúdo reflexivo permite explicar a mudança na situação de Gary (de Mary) quando ele (ela) deixa a cabana sem janelas (o quarto preto e branco) e vê Little America (o tomate maduro) não como a apreensão de um novo fato sobre o mundo, mas como uma mudança na sua relação reflexiva com os fatos. Por isso, a posição defendida por Perry pode ser descrita como uma forma de materialismo contextualista reflexivista.

O ponto crucial para Perry é que o argumento do conhecimento só nos leva a considerar como obrigatória a expansão ontológica além das propriedades e dos fatos previamente conhecidos quando cometemos a subject matter fallacy, isto é, quando aceitamos sem questionar a subject matter assumption: a tese segundo a qual o conteúdo referencial é todo o conteúdo que existe. Essa assunção se revela falha nos casos ordinários de conhecimento recognicional. O que vale para Gary pode valer para Mary, em particular, e o conhecimento fenomenal, em geral. Se o novo conhecimento de Mary for um caso de conhecimento recognicional, então ele não requer a expansão da ontologia além do âmbito dos fatos físicos. Isto não mostra que o conhecimento fenomenal é a aquisição de informação física. O que é certo é que a analogia proposta por Perry entre o conhecimento fenomenal e o conhecimento recognicional bloqueia a conclusão dualista do argumento do conhecimento. Se não temos de aceitar o dualismo, podemos continuar firmes na nossa crença fisicista.

\section{V}

A visão de Perry parece implicar que o conceito mobilizado no novo conhecimento de Mary é um conceito indexical ou demonstrativo. Isto se reflete na expressão do conhecimento fenomenal. Se a descoberta de Gary podia ser expressa por

(11) Este lugar é Little America,

o novo conhecimento de Mary pode ser expresso, de acordo com Perry, por

(13) Isto $_{i}$ é o que é ter a experiência de vermelho.

Pelo menos aparentemente, as palavras "este lugar" e "isto $i$ " funcionam aqui como expressões de conceitos indexicais ou demonstrativos genuínos. Este lugar é, sem dúvida, o conceito demonstrativo de um lugar particular. A analogia entre (11) e (13) sugere que isto $_{i}$, por sua vez, também seria um conceito demonstrativo. Obviamente, isto $_{i}$ não pode ser visto como o conceito demonstrativo de um particular, mas de um universal, de um tipo de estado fenomenal ou experiência que Mary tem 
pela primeira vez quando sai do quarto e que seres humanos normais sempre têm (sempre tiveram) quando se encontram (se encontravam) na situação epistêmica de Mary. Perry concordaria, portanto, com vários outros filósofos (por exemplo, Loar), para os quais os conceitos fenomenais são "conceitos demonstrativos de tipo" (typedemonstrative concepts).

Uma objeção à teoria de Perry, em particular, ou a qualquer teoria que parta da analogia entre o conhecimento fenomenal e o conhecimento indexical, em geral, questionaria a interpretação dos conceitos fenomenais como demonstrativos. De fato, David Papineau (2006, p. 113 e seg.) pensa que a interpretação demonstrativa dos conceitos fenomenais erra ao não dissociar expressão linguística e conceito expresso. A não-percepção do caráter imperfeito da analogia entre (11) e (13) poderia induzir ao erro. O conceito este lugar identifica o seu objeto via um modo indexical de apresentação. O objeto, nesse caso, é um lugar particular para o qual aponta demonstrativamente o gesto indicativo que acompanha o proferimento de "este lugar". Aqui há adequação entre conceito (demonstrativo) e expressão linguística (indexical). O objeto do conceito fenomenal expresso por "isto ${ }_{i}$ ", por sua vez, não é uma experiência particular, mas um tipo de experiência. Na visão de Papineau, isto impede que associemos ao conceito fenomenal um modo indexical de apresentação como link entre o conceito fenomenal e seu objeto. Do ponto de vista de Papineau, é razoável pensar que a indicação demonstrativa é restrita aos objetos particulares. Por certo, é possível apontar para as instanciações particulares dos universais, mas não para os universais em si mesmos. Se ainda assim quisermos recorrer a uma palavra indexical como "isto" para expressar um conceito fenomenal, então devemos reconhecer que existe, nesse caso, uma inadequação básica entre conceito e expressão linguística. A expressão é indexical, mas o conceito expresso não é demonstrativo.

A característica essencial/definitória de termos indexicais ou demonstrativos é a dependência do valor referencial de tais termos vis-à-vis diferentes contextos de uso. A teoria semântica que se tornou consensual associa a termos demonstrativos um "caráter" kaplaniano que desempenha o papel de fixador da referência. O caráter kaplaniano é uma forma de significado, parte central de uma função que leva de contextos de uso a referentes. O significado de "este lugar", como em (11), por exemplo, pode ser dado por "este lugar, qualquer que ele seja". O ponto importante é que caráter de termos indexicais deixa em aberto a questão sobre o seu referente. Esta só é fechada pela informação sobre o contexto de uso. No contexto de Gary (o proferidor de (11) "Este lugar é Little America"), o referente é Little America - e (11) expressa uma verdade. Em outro contexto, no qual Gary poderia estar, o referente é diferente - e (11) expressaria uma falsidade. Em virtude da adequação entre conceito e expressão, é possível associar também ao conceito expresso este lugar um caráter que deixa em aberto a questão sobre o seu objeto. Da mesma forma, o conceito esse pássaro, mobilizado na indicação demonstrativa de um pássaro particu- 
lar, designa diferentes pássaros em diferentes contextos. Mas o valor referencial do conceito esse (tipo) de pássaro, ao contrário, não varia de contexto para contexto. $\mathrm{O}$ que pode variar é, plausivelmente, o pássaro particular que exemplifica o tipo (contextualmente não-variável) de pássaro intencionado. Como Papineau diz, o conceito de um tipo de objeto, em oposição ao conceito de um objeto particular, não apresenta characterlikeness. Como é possível — talvez seja inevitável — usar a locução "esse pássaro", tipicamente dotada de caráter, como expressão do conceito de um tipo de pássaro - um conceito que não exibe caráter — , nós devemos distinguir conceito e expressão. O resultado líquido é o mesmo do último parágrafo: o conceito é não-demonstrativo, ainda que as palavras que o expressam sejam.

Isto deve valer a fortiori também para os conceitos fenomenais. "Esta experiência" pode apontar para o estado fenomenal particular em que Mary se encontra ao sair do quarto. Mas o que é relevante para o argumento do conhecimento é o tipo de experiência que Mary adquire agora, o mesmo tipo de estado fenomenal que seres humanos normais instanciam quando confrontados com tomates vermelhos, por exemplo. O termo "isto ${ }_{i}$ " tal como aparece em (13) "Isto ${ }_{i}$ é o que é ter a experiência de vermelho" tem, portanto, o sentido de "este tipo de experiência, qualquer que ele seja". Contudo, se Papineau estiver certo, o conceito correspondente não exibe characterlikeness. A crítica pode ser posta na forma de um dilema. Se o indexical "isto ${ }_{i}$ " expressar de fato um conceito demonstrativo, este conceito não é de um tipo de experiência. E se o conceito for de um tipo de experiência, então ele não será demonstrativo - e o uso de "isto ${ }_{i}$ " deve ser visto como um expediente comunicativo enganador na falta de uma expressão mais adequada.

\section{VI}

Há pelo menos duas possibilidades relevantes para a defesa do ponto de vista contextualista reflexivista de Perry frente à critica de Papineau. A primeira parte da observação de que o caráter problemático apontado por Papineau na noção de conceito demonstrativo de tipo deriva da compreensão restritiva do universo das entidades que podem ser indicadas demonstrativamente. A restrição é tal que somente particulares, mas não universais (tipos, propriedades, relações etc.) podem figurar como referentes de conceitos demonstrativos. Se apontar para tipos de objetos for, em última instância, apontar para os objetos particulares desse tipo, então não é possível que outras entidades que não particulares sejam identificadas (picked out) via modos indexicais de apresentação. Os conceitos demonstrativos seriam, portanto, necessariamente, conceitos de particulares. Isto se reflete na tentativa de associar a conceitos de tipos um caráter kaplaniano que deixe em aberto os seus referentes. Os contextos de uso correspondentes são especificados em termos das entidades que 
definem a situação real em que a expressão do conceito é proferida. Ao restringir, com Papineau, essas entidades aos particulares, nós não conseguiremos mostrar que um conceito de tipo qualquer denote diferentes itens em diferentes contextos. O que é variável nos contextos de uso especificados dessa maneira é o objeto particular que exemplifica o tipo em questão, mas este não é o referente do conceito. O conceito de tipo não denota um objeto particular em uma situação de uso e um objeto particular diferente em outra situação. Ele denota o mesmo tipo exemplificado por diferentes objetos particulares em diferentes contextos de uso especificados à la Papineau.

$\mathrm{O}$ argumento de Papineau contra a interpretação de conceitos fenomenais como demonstrativos depende, portanto, de uma assunção relativamente implícita sobre quão indireta é a nossa relação cognitiva com os tipos ou as propriedades, quando comparada com a nossa relação com os particulares que instanciam estas propriedades. Como se antecipasse a objeção de Papineau, Perry considera (e rejeita) a assunção implícita na argumentação exposta na seção anterior. Do ponto de vista de Perry, há duas posições extremas. Uma considera que o conhecimento dos universais (tipos, propriedades) é mais direto que a relação com particulares e que os objetos particulares são conhecidos pelas propriedades que eles instanciam. De acordo com a outra posição extrema, os universais são "menos reais" do que os particulares: o conhecimento é fundamentalmente de particulares e é somente via alguma forma de abstração que os universais podem ser conhecidos. Perry se decide por uma posição intermediária. "I shall assume that universals are real, or at least as real as particulars [...] and that in standard case they are known via sensations and concepts" (Perry 2001, p. 140). Embora isso não possa ser considerado a demonstração racional da falsidade da assunção implícita no argumento de Papineau, há aqui pelo menos a indicação, reconhecidamente vaga, de uma posição não obviamente implausível - e talvez defensável — que permitiria associar aos conceitos de tipos fenomenais um núcleo demonstrativo genuíno. Se, além de experiências particulares, tipos de experiência também puderem figurar na especificação dos contextos de uso de conceitos fenomenais, então os referentes de tais conceitos (os tipos fenomenais) apresentarão a mesma variação contextual que é própria dos conceitos demonstrativos não-controversos, como este lugar. Em outras palavras, os conceitos fenomenais apresentarão, em um plano mais elevado, um caráter kaplaniano que reflete a dependência contextual dos seus objetos, isto é, um caráter semelhante ao exibido, em um plano menos elevado, pelos conceitos demonstrativos não-controversos. Uma das vantagens desta resposta à crítica de Papineau é que a expressão linguística ordinária e bem estabelecida dos conceitos fenomenais não precisa mais ser vista como essencialmente enganadora.

A alternativa para a defesa do ponto de vista contextualista reflexivista de Perry frente à critica de Papineau consiste, em primeiro lugar, em conceder o diagnóstico negativo sobre a suposta natureza demonstrativa dos conceitos fenomenais, afir- 
mando, ao mesmo tempo, a irrelevância do diagnóstico para a posição mais geral defendida por Perry. O que é importante na resposta contextualista reflexivista de Perry ao argumento do conhecimento é a demonstração de que o conteúdo do conhecimento fenomenal é sempre uma informação essencialmente dependente de contexto. Isto pressupõe que existem informações de tal forma ligadas a um contexto que elas só podem ser adquiridas por quem se encontra nesse contexto. Em particular, se o novo conhecimento de Mary for desse tipo, nada impede que a nova informação adquirida por ela seja física, ao contrário do que é sugerido pelo argumento do conhecimento. Evidentemente, a dependência contextual do conteúdo do conhecimento fenomenal estará imediatamente assegurada se os conceitos fenomenais que compõem o conteúdo fenomenal forem demonstrativos: a dependência contextual dos constituintes demonstrativos seria automaticamente transferida aos conteúdos constituídos. De fato, porém, a dependência contextual do conhecimento fenomenal é assegurada na teoria de Perry mediante a distinção entre conteúdo referencial e conteúdo reflexivo. Esta distinção é neutra relativamente à questão sobre caráter demonstrativo dos conceitos fenomenais. Ela não é aplicada exclusivamente a estados mentais em cuja expressão aparecem termos indexicais. Isto quer dizer que Perry poderia (i) substituir, na expressão do novo conhecimento de Mary, o termo "isto ${ }_{i}$ " por um termo genérico qualquer, por exemplo, " $R$ ", (ii) conceder a Papineau que $R$ é um conceito geral, não-demonstrativo e, ainda assim, (iii) insistir que é possível atribuir um conteúdo referencial $e$ um conteúdo reflexivo diferente também ao estado mental correspondente à nova expressão modificada. Isto levaria à consideração de

(14) $R$ é o que é ter a experiência de vermelho

como expressão de um conhecimento fenomenal que não é constituído por um conceito demonstrativo. (14) tem um conteúdo referencial que já tinha sido apreendido por Mary antes de sair do quarto preto e branco. O conhecimento prévio de Mary inclui a informação referencial de que objetos vermelhos causam, em circunstâncias internas e externas normais, experiências do tipo $R$, pois essa informação pode ser adquirida mesmo por quem não tem a experiência correspondente. ( $\mathrm{Na}$ fonte da informação, evidentemente, estão pessoas que passaram pela experiência correspondente.) O que é novo para Mary, o que Mary poderia expressar também com (14), é o conteúdo reflexivo: a proposição que é verdadeira se e somente se as experiências resultantes do confronto normal com objetos vermelhos forem do tipo designado pelo uso de " $R$ " por parte daquela que profere (14). Há aqui um aspecto de dependência contextual mais ou menos óbvio. Mas ele não está ligado ao caráter indexical da expressão " $R$ " ou ao aspecto demonstrativo do conceito $R$. A concessão feita à critica de Papineau é que o termo " $R$ " não é um indexical, nem contém um componente indexical oculto, podendo ser visto como expressão adequada do conceito 
não-demonstrativo $R$. A dependência contextual do novo conhecimento de Mary poderia ser assegurada pela natureza demonstrativa do conceito fenomenal subjacente, se este fosse de fato um conceito demonstrativo. Mas ela pode ser assegurada - ou melhor: ela é assegurada - na teoria de Perry pela reflexividade que o novo conhecimento de Mary partilha com as outras formas de conhecimento recognicional. Isto quer dizer que o contextualismo reflexivista de Perry pode prescindir da visão criticada por Papineau, segundo a qual conceitos fenomenais são demonstrativos de tipo.

\section{VII}

De modo semelhante a Papineau, Chalmers também pensa que os conceitos fenomenais mais importantes não estão ligados aos seus objetos via um modo indexical de apresentação. Embora reconheça, ao contrário de Papineau, a existência de um elemento indexical/demonstrativo entre os conceitos mobilizados por Mary ao sair do quarto, Chalmers defende que o conteúdo do conhecimento verdadeiramente relevante para o argumento do conhecimento não tem qualquer traço de indexicalidade. De acordo com Chalmers, o conhecimento indexical irrelevante envolvido no caso de Mary é o que poderia ser expresso por:

(15) Isto é o que é ter a experiência de vermelho, ao passo que o novo conhecimento crucial de Mary é o que seria expresso por:

(16) Objetos vermelhos causam experiências $R$,

onde $R$, em oposição a isto, deve ser interpretado como um conceito "qualitativo" e isso quer dizer, entre outras coisas: um conceito radicalmente não-demonstrativo - do tipo de experiência que Mary está tendo agora. (15) e (16) expressariam, portanto, cognições de diferentes conteúdos envolvendo conceitos aos quais são associados diferentes modos de apresentação: um modo indexical de apresentação, por um lado, e um modo "qualitativo" de apresentação, por outro. Sob essas condições relativamente estipulativas, o que quer que Perry possa dizer de esclarecedor sobre a dependência contextual ou a natureza demonstrativa do conhecimento fenomenal não pode ser transportado para a análise do novo conhecimento importante para o argumento do conhecimento:

I conclude that Perry gives an adequate analysis of certain subsidiary demonstrative knowledge that Mary gains-knowledge that is analogous to demonstrative knowledge in other domains. But Perry gives no adequate analysis of Mary's substantive new non-indexical, non-demonstrative phenomenal knowledge, and in particular gives no way of reconciling this new phenomenal knowledge with the truth of physicalism (Chalmers 2004, p. 186).

Principia 15(3): 415-438 (2011). 
Isso está de acordo com a hierarquia de conceitos fenomenais afirmada por Chalmers em outro trabalho:

When one believes that one is having a red experience, one deploys a phenomenal concept of a red experience. The most important phenomenal concepts are those that we acquire directly from having experiences with that sort of phenomenal character. (Chalmers 2010, p. 251)

Portanto, mesmo sob a suposição de que existem conceitos fenomenais que identificam indexicalmente propriedades fenomenais - o que é enfaticamente negado por Papineau - eles não estariam, quase que por definição, entre os conceitos fenomenais mais importantes. Igualmente excluídos do âmbito dos conceitos fenomenais "puros" ou "diretos" de Chalmers estão aqueles conceitos fenomenais cujos referentes são fixados via as relações a objetos paradigmáticos que são indicados ostensivamente no aprendizado da linguagem pública. O significado do conceito fenomenal expresso pela palavra "vermelho", por exemplo, pode ser dado por: "a qualidade fenomenal tipicamente causada em sujeitos normais na minha comunidade por objetos paradigmaticamente vermelhos" (Chalmers 2010, p. 255). Dito em termos menos informais: o "conceito relacional comunitário vermelho ${ }_{C}$ " não é um conceito fenomenal puro. Tampouco é puro o "conceito relacional individual vermelho ${ }_{I}$ " - cuja existência não é posta em questão por Chalmers. Este é o conceito que identifica "a qualidade fenomenal tipicamente causada em mim por objetos paradigmaticamente vermelhos". Chalmers recorre a estas considerações para determinar de modo contrastivo o que são os conceitos fenomenais puros:

The three concepts red $_{C}$, red $d_{I}$ and this $s_{E}$ may all refer to the same quality, phenomenal redness. In each case, reference is fixed relationally, with the quality characterized in terms of its relations to external objects or acts of ostension. There is another crucial concept in the vicinity, one that does not pick out phenomenal redness relationally but rather picks it out directly in terms of its intrinsic phenomenal nature. This is what we might call a pure phenomenal concept. (Chalmers 2010, p. 256)

Como outros defensores dos conceitos fenomenais, Chalmers não ignora que há um preço a pagar pela afirmação de que existe um tipo de conceito cujo referente está presente no seu sentido (sense) "de um modo que é mais forte do que no caso usual da referência direta" (Chalmers 2003, p. 233). Conceitos fenomenais puros são essencialmente privados e, portanto, de difícil expressão na linguagem comum (Chalmers 2010, p. 258). Eles são não-deferenciais, no sentido em que sua aplicação, ao contrário do que ocorre com os conceitos ordinários como artrite ou estrela, mas também red r ou this $_{E}$ - não é controlada pelo que os membros mais competentes da minha comunidade linguística dizem sobre o que são os seus referentes. Conceitos fenomenais puros, uma vez adquiridos, não podem ser "armazenados na memória" 
para uso posterior. Eles têm de ser adquiridos diretamente da mesma experiência à qual eles são aplicados (Chalmers 2010, p. 268). E eles têm de ser readquiridos a cada nova experiência:

The lifetime of a direct phenomenal concept is limited to the lifetime of the experience (or the instantiated quality) that constitutes it. (In some cases a specific phenomenal concept might persist for a few moments due to the persistence of a vivid iconic memory, but even this will soon disappear.) (Chalmers 2010, p. 272)

Papineau (2006, p. 111) observa que os conceitos fenomenais especiais são invocados por quase todos os filósofos contemporâneos que propõem teorias sobre a mente consciente, sejam eles materialistas ou anti-materialistas. Nem todos os filósofos endossariam todos os traços que Chalmers considera centrais para os conceitos fenomenais puros, mas qualquer um deles endossa muitos traços. Esta constatação é intrigante, pois, independentemente da questão sobre se Chalmers ou qualquer outro consegue ou não dar substância a essa noção altamente controversa de conceito fenomenal puro, ela é irrelevante para o problema maior discutido na filosofia da mente consciente. O problema era o de determinar se a suposição de que Mary adquire um novo conhecimento proposicional é compatível ou incompatível com a verdade do fisicismo. O objeto do conhecimento em torno do qual gira o argumento do conhecimento tem a ver com as propriedades das experiências de seres humanos normais, não com propriedades que constituiriam o mundo privado e publicamente inacessível de Mary. O ponto é claramente afirmado por Jackson:

[T] he knowledge Mary lacked which is of particular point for the knowledge argument against physicalism is knowledge about the experience of others, not about her own. (Jackson 1986, p. 293)

Mas isto implica que o novo conhecimento de Mary, na medida em que é sobre as experiências de outros, deve necessariamente envolver conceitos "impuros", relacionais, deferenciais, públicos e persistentes, conceitos que Mary deve necessariamente ter armazenado na memória antes mesmo de ter a sua primeira visual de objetos vermelhos. Pode-se conceder que Mary tinha uma compreensão apenas parcial do conceito fenomenal que ela aplica quando sai do quarto preto e branco que a compreensão completa vem somente a partir das novas experiências. Todavia, a compreensão parcial basta para que Mary já tenha, durante o seu confinamento, os conceitos "impuros" necessários para a aquisição do conhecimento sobre as experiências visuais de outros seres humanos além dela mesma (cf. Ball 2009 e Tye 2009). Mais uma vez, é Perry que expõe as coisas de forma convincente:

So let's assume that Mary does have a concept of [the subjective character that people with normal vision have when they look at a red object] that is

Principia 15(3): 415-438 (2011). 
actually quite rich. It contains a number of things that mine does, such as being the subjective character of normal red experiences, but a great deal more that she has gleaned from her textbooks. It doesn't, however, contain any Humean ideas of color impressions, any memory images. Her own experiences have not been a direct source of information for these concepts of color sensations, and the concepts have never been applied to color sensations of her own. (Perry 2001, p. 99)

Neste ponto, a "estratégia dos conceitos fenomenais" começa a aparecer sob uma luz bastante desfavorável. Como já comentado na primeira seção, a idéia motora desta estratégia era mostrar (i) que existe um hiato epistêmico entre o domínio dos processos físicos e o das experiências subjetivas, (ii) que as peculiaridades dos conceitos fenomenais explicam (tornam "justificada") a intuição negativa comum que emerge imediatamente da mera consideração da identidade de propriedades fenomenais e propriedades físicas, mas (iii) que o dualista comete um erro ao inferir um hiato ontológico do hiato epistêmico. Em particular, os conceitos fenomenais deveriam fornecer a chave para a compatibilização do fisicismo com a suposição de que Mary adquire um novo conhecimento proposicional ao sair do quarto preto e branco. As considerações feitas na presente seção parecem indicar que ou bem os conceitos fenomenais são especiais, mas inúteis para a discussão do caso de Mary e do conhecimento geral que ela supostamente adquire, ou bem os conceitos fenomenais são tão relacionais, deferenciais, públicos e persistentes quanto os conceitos ordinários - e nesse caso o materialista precisa encontrar um outro ponto de apoio para resistir ao argumento do conhecimento.

\section{VIII}

O materialismo contextualista reflexivista de Perry vê a solução do problema na dependência contextual essencial do conhecimento fenomenal. Isso envolve a consideração de casos de conhecimento fenomenal como conhecimento recognicional e a distinção entre o conteúdo referencial e o conteúdo reflexivo das representações mentais de Mary. O conteúdo referencial é completamente especificado em termos das entidades que constituem o subject matter das representações mentais de Mary, isto é, em termos das condições que a verdade da crença de Mary impõe às entidades objetivas (os objetos e as propriedades) a que se referem os componentes do seu estado de crença (belief state). Ao descrever na perspectiva da terceira pessoa as possibilidades objetivas - ao descrever como o mundo objetivo "externo" a Mary deveria ser - para que a representação de Mary seja verdadeira, nós estaríamos especificando o que Mary pensa (o conteúdo de seu estado mental). O conteúdo reflexivo, por outro lado, incorpora os aspectos da perspectiva subjetiva de Mary sobre 
o mundo objetivo, o modo como ela representa os fatos objetivos. Isso permite caracterizar a transformação pela qual Mary passa quando sai do confinamento não como a apreensão de um novo fato, mas como uma mudança na sua relação cognitiva com os mesmos fatos que ela já tinha apreendido antes, sob outro modo de apresentação. A dependência contextual essencial do conhecimento fenomenal, em particular, e do conhecimento recognicional, em geral, é localizada, portanto, no nível do conteúdo reflexivo.

Do ponto de vista do materialismo contextualista não-reflexivista de Stalnaker, ao contrário, é possível dar conta dos fatos significativos que definem o ponto de vista subjetivo de quem representa recognicionalmente o mundo no quadro de uma teoria que descreve "de fora" as possibilidades objetivas incluídas (e as possibilidades objetivas excluídas) pela verdade da representação em questão. Stalnaker propõe a reconstrução da noção de conteúdo representacional ou informacional de tal forma que este absorve, por assim dizer, o núcleo da distinção feita por Perry entre conteúdo referencial e conteúdo reflexivo. Com isso, a dependência contextual essencial é retirada do plano dos modos de apresentação dos fatos do mundo e localizada no plano das possibilidades relevantes que entram na especificação do conteúdo informacional, isto é, no plano dos próprios fatos do mundo.

$\mathrm{Na}$ visão de Stalnaker, o conteúdo informacional do conhecimento recognicional é um tipo de conteúdo referencial (embora não exatamente tradicional), isto é, uma proposição determinada pelas condições que a verdade da crença correspondente impõe aos referentes dos componentes do estado de crença, sem qualquer referência "reflexiva" aos elementos da representação. Conteúdos são atribuídos por Stalnaker, em primeiro lugar a asserções, atos de fala que transmitem informação sobre o mundo. Estados mentais herdam, por assim dizer, o conteúdo dos atos de fala que os expressam. As condições de verdade que determinam o conteúdo de uma asserção podem ser representadas pelo conjunto das situações objetivas possíveis onde a asserção é verdadeira e das situações objetivas possíveis onde a asserção é falsa. As possibilidades envolvidas na representação do conteúdo não são todas as possibilidades. Considerações pragmáticas excluem como irrelevantes, em cada caso, possibilidades que não entram na especificação do conteúdo. O conjunto das possibilidades relevantes define o que Stalnaker chama de "conjunto-contexto" (context set). Tais possibilidades são as possibilidades compatíveis com tudo que os participantes do jogo de linguagem da asserção sabem — ou pensam que sabem — até o ponto em que algo novo seja asserido. Uma asserção pode ser vista, portanto, como uma proposta de partição no conjunto das possibilidades. O efeito essencial da asserção - se ela for aceita pelos participantes da conversação como verdadeira é reduzir o conjunto de possibilidades, redefinir o conjunto-contexto que servirá de base à interpretação de novas asserções. Se a asserção for, por exemplo, "O trem parte às nove horas" e as possibilidades relevantes puderem ser caracterizadas por

Principia 15(3): 415-438 (2011). 
(i) "partida do trem às sete horas", $(j)$ "partida do trem às oito horas", e $(k)$ "partida do trem às nove horas" - possibilidades abstrusas como "o trem explodiu" são pragmaticamente excluídas - então o conteúdo pode ser representado pela seguinte tabela:

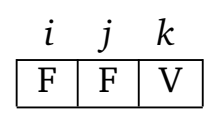

A matriz acima reflete um tipo de dependência do conteúdo vis-à-vis os fatos: os fatos determinam se o que foi asserido é verdadeiro ou falso. Há, contudo, uma outra forma de dependência que se manifesta claramente nos casos de conhecimento recognicional: os fatos determinam o que é dito. Considere, por exemplo, a asserção de Gary quando ele sai do quarto:

(11) Este lugar é Little America.

Considere, além disso, que Gary sabe que está na frente de um posto de gasolina e que só há três postos de gasolina na estrada: (i) Little America, ( $j$ ) Little Asia, (k) Little Europe. Nessas condições, as regras semânticas tradicionalmente aceitas levam a atribuição de um conteúdo proposicional à sentença proferida por Gary, como o conteúdo expresso pela sentença. Esta proposição depende evidentemente do contexto onde ela é proferida. Isto leva a seguinte matriz, que Stalnaker chama de "conceito proposicional" da asserção (14):

\begin{tabular}{l|l|l|l|}
\multicolumn{1}{c}{} & \multicolumn{1}{c}{$i$} & $j$ & $k$ \\
\cline { 2 - 4 }$i$ & $\mathrm{~V}$ & $\mathrm{~V}$ & $\mathrm{~V}$ \\
\cline { 2 - 4 }$j$ & $\mathrm{~F}$ & $\mathrm{~F}$ & $\mathrm{~F}$ \\
\cline { 2 - 4 }$k$ & $\mathrm{~F}$ & $\mathrm{~F}$ & $\mathrm{~F}$ \\
\cline { 2 - 4 } & &
\end{tabular}

Na vertical estão representadas as situações possíveis consideradas como contextos de proferimento. Na horizontal as situações possíveis aparecem como circunstâncias de avaliação. As regras semânticas tradicionalmente aceitas tratam "Este lugar" e "Little America" como designadores rígidos. Isto quer dizer que a proposição semanticamente expressa por (11) será uma proposição necessária: necessariamente verdadeira, se o proferimento de (11) ocorrer em $i$; necessariamente falsa, se o proferimento de (11) ocorrer em $j$; necessariamente falsa, se o proferimento de (11) ocorrer em $k$. Estas proposições, representadas nas linhas da matriz acima, não podem ser a informação que Gary está transmitindo quando assere (11). Intuitivamente, Gary está comunicando ou tentando comunicar algo contingente. Intuitivamente, ao asserir (11), Gary está excluindo a possibilidade de que ele esteja em frente a Little Asia ou Little Europe. A informação contingente que ele está transmitindo é verdadeira em $i$, falsa em $j$ e falsa em $k$. Esta informação está representada 
na diagonal (de cima para baixo, da esquerda para a direita) do conceito proposicional construído acima.

Como ocorre com as proposições semanticamente expressas pela sentença asserida (as linhas do conceito proposicional correspondente), o conteúdo informativo de uma asserção (a proposição diagonal) também é puramente referencial. Ele propõe a exclusão de algumas possibilidades do meio ambiente onde se situa o sujeito da asserção.

Aplicada, agora, ao caso de Mary, a estratégia de Stalnaker considera que algo acontece em Mary (presumivelmente no seu cérebro) quando ela sai do quarto: a instanciação de uma propriedade física objetiva que o seu estado mental atual tem, independente das relações com outros estados e eventos mentais ou não mentais. Ao asserir

(13) Isto $_{i}$ é o que é ter a experiência de vermelho,

Mary exclui algumas possibilidades objetivas. O que é distintivo do conhecimento fenomenal de Mary é que o conjunto-contexto inclui possibilidades que só são live options para quem está adequadamente situado frente ao que acontece com Mary quando ela tem a sua nova experiência. E, evidentemente, Mary é a única pessoa que está no "lugar certo": somente Mary tem acesso fenomenal aos seus estados mentais - somente Mary está apropriadamente conectada ao seu cérebro. $\mathrm{O}$ característico do conhecimento fenomenal não é que ele seja a apreensão de propriedades nãofísicas, mas que a informação fenomenal é essencialmente dependente do contexto onde ela é adquirida.

A dependência contextual não é exclusividade do conhecimento fenomenal. Ela já aparece no caso de Gary descrito acima. As opções relevantes para Gary são (i) "Este lugar é Little America", (j) "Este lugar é Little Asia", (k) "Este lugar é Little Europe". Se as possibilidades fossem redescritas num contexto mais amplo — por exemplo, em termos das coordenadas exatas dos pontos da estrada onde estão situados Little America, Little Asia e Little Europe - a informação poderia adquirir um caráter mais descontextualizado. Mas, intuitivamente pelo menos, a informação que Gary está tentando transmitir não é sobre as coordenadas exatas "neutras" do local onde ele se encontra. O contexto constituído pelas possibilidades (i) "Este lugar é Little America", ( $j$ ) "Este lugar é Little Asia" e $(k)$ "Este lugar é Little Europe" parece ser essencial à identidade da informação veiculada por Gary. E isso se aplica também para a informação veiculada por Mary.

\section{IX}

As respostas materialistas contextualistas de Perry e Stalnaker fornecem explicações plausíveis para os casos de conhecimento recognicional. Se estas explicações pude-

Principia 15(3): 415-438 (2011). 
rem ser de fato estendidas ao caso do conhecimento fenomenal, então o materialismo contextualista responde ao desafio do argumento do conhecimento. Ele não mostra que os fatos apreendidos fenomenalmente são fatos físicos. A conclusão é mais modesta. Ele diz somente que o argumento do conhecimento não mostra que o conhecimento fenomenal é a aquisição de informação sobre a realidade não-física. Evidentemente, o poder de convencimento deste resultado depende inteiramente do antecedente do condicional: se o conhecimento fenomenal puder ser analisado como conhecimento recognicional, então o caso de Mary não é um problema para o materialista.

Contra qualquer proposta de redução do conhecimento fenomenal ao conhecimento recognicional, Chalmers propõe um argumento direto. O ponto de partida da crítica de Chalmers é a observação de que o estado de ignorância típico dos que se encontram na situação pré-recognicional não é partilhado por um observador fisicamente onisciente:

Say that I am physically omniscient, but do not know whether I am in the USA or Australia (let's imagine that there are appropriate qualitative twins in both). Then I have a certain indexical ignorance, and discovering that I am in the USA will constitute new knowledge. But if someone else is watching from the third-person point of view and is also physically omniscient, they will have no corresponding ignorance: they will know that $A$ is in Australia and that $B$ is in the USA, and that's that. (Chalmers 2004, p. $186 \mathrm{e}$ seg.)

Como o fenômeno do desaparecimento da ignorância não se manifesta no caso do conhecimento fenomenal, Chalmers conclui que a analogia mobilizada na resposta contextualista ao argumento do conhecimento está simplesmente errada.

Now consider Mary's ignorance. From her black-and-white room, she is ignorant of all sorts of facts: what it will be like for her to see red for the first time, what it is like for others to see red, and so on. [...] a physically omniscient observer may have precisely analogous ignorance: even given his complete physical knowledge, he may have no idea what it will be like for Mary to see red for the first time. So this ignorance does not evaporate from the objective viewpoint. The same goes even more strongly for knowledge of what it is like for others to see red. For any observer, regardless of their viewpoint, there will be an epistemic gap between complete physical knowledge and this sort of phenomenal knowledge. This suggests very strongly that phenomenal knowledge is not a variety of indexical or demonstrative knowledge at all. (Chalmers 2004, p. 187)

$\mathrm{Na}$ avaliação desse argumento, é importante considerar o fato de que a dependência contextual do conteúdo do conhecimento recognicional é, do ponto de vista do materialista contextualista, uma questão de grau. Stalnaker chama atenção para 
o fato de que a distinção entre informação essencialmente contextual e informação independente do contexto não deve ser compreendida como uma dicotomia. O que existe é um continuum, um espectro que tem, numa das extremidades, informações essencialmente contextuais e, na outra extremidade, informações muito descontextualizadas, como, por exemplo, "Baleias não são peixes" e "Água ferve a cem graus, em condições normais de pressão". O caso do agente imaginado por Chalmers, que não sabe se está na Austrália ou nos EUA, é o caso de conteúdo informacional relativamente descontextualizado. A ausência da ignorância correspondente no observador externo fisicamente onisciente é a medida da descontextualização. Da mesma forma, o fato de que a observação mais completa dos processos físicos ainda não nos dá uma idéia do que Mary aprende quando sai do seu confinamento, mostra somente que o caso de Mary e do conhecimento fenomenal, por sua vez, deve estar bem mais próximo da extremidade das informações essencialmente contextuais.

Para o materialista contextualista, o que faz de um conteúdo informacional qualquer um conteúdo essencialmente contextual é exatamente o fato de que ele não pode ser apreendido por qualquer outra pessoa situada fora do contexto relevante, mesmo que ela já tenha adquirido todos os fatos descontextualizados. Isto não é um expediente ad hoc inventado para dar conta dos casos de conhecimento fenomenal. Casos de conhecimento não-fenomenal também podem ser essencialmente contextuais. Após introduzir o caso de Alice - que adquire conhecimento recognicional de que a bomba localizada aqui vai explodir daqui a meia hora - e Barry - que é onisciente sobre os fatos que Alice vai experimentar diretamente - , Stalnaker diz: "Barry knows a lot about the situation, in this little story, but he doesn't know what Alice will know the next day. He is not in a position to know this; he would have to be there" (Stalnaker 2008, p. 85).

Portanto, mesmo assumindo, com Chalmers, que a lacuna epistêmica permanece no caso do conhecimento fenomenal e do observador fisicamente onisciente, mas desaparece no caso do agente que não sabe em que país está e do correspondente observador externo, o materialista contextualista não está racionalmente obrigado a aceitar que a analogia entre o conhecimento fenomenal e o conhecimento recognicional está errada. A conclusão razoável, mais uma vez, é que não existe um argumento que revele uma falha comprometedora na resposta materialista contextualista ao desafio representado pelo argumento do conhecimento.

\section{References}

Alter, T. e S. Walter (orgs.) 2006. Phenomenal Concepts and Phenomenal Knowledge. New Essays on Consciousness and Physicalism. Oxford: Oxford University Press.

Ball, D. (2009) There Are No Phenomenal Concepts. Mind 118: 935-62.

Principia 15(3): 415-438 (2011). 
Block, N., O. Flanagan e G. Güzeldere (orgs.) 1997. The Nature of Consciousness. Cambridge, MA: The MIT Press.

Chalmers, D. 1996. The Conscious Mind. Oxford: Oxford University Press.

—. 2003. Consciousness and its Place in Nature. Em Stich e Warfield (orgs.) 2003, p. $102-42$.

- 2004. Imagination, Indexicality, and Intensions. Disponível em: http://consc.net/papers/perry.html.

- 2005. Phenomenal Concepts and the Knowledge Argument. Em Ludlow, Nagasawa e Stoljar 2005, p. 269-98.

- 2010. The Character of Consciousness. Oxford: Oxford University Press.

Goodman, N. 1951. The Structure of Appearance. Dordrecht: D. Reidel.

Jackson, F. 1982. Epiphenomenal Qualia. Philosophical Quarterly 32: 127-36.

- 1986. What Mary Didn't Know. Journal of Philosophy 83: 291-95.

Levin, J. 2007. What is a Phenomenal Concept? Em Alter e Walter 2006, p. 87-110.

Loar, B. 1990. Phenomenal States. Philosophical Perspectives 4: 81-108.

_ 1997. Phenomenal States (Revised Version). Em Block, Flanagan e Güzeldere 1997, p. 597-616.

Ludlow, P., Y. Nagasawa, e D. Stoljar (orgs.) 2005. There is something about Mary: essays on phenomenal consciousness and Frank Jackson's knowledge argument. Cambridge, MA: The MIT Press.

Papineau, D. 2002. Thinking about Consciousness. Oxford: Oxford University Press.

- 2006. Phenomenal and perceptual concepts. Em Alter e Walter 2006, p. 111-44.

Perry, J. 1979. The Problem of the Essential Indexical. Nous 13: 3-21.

- 2001. Knowledge, Possibility and Consciousness. Cambridge, MA: The MIT Press.

- 2004. Replies. Philosophical and Phenomenological Research 68: 207-29.

- 2006. Stalnaker and Indexical Belief. Em Thomson e Byrne 2006, p. 204-21.

Stalnaker, R. 1978. Assertion. Em Cole 1978, p. 315-32. (Reimpresso em Stalnaker 1999, p. 78-95.)

-1999. Content and Context. Oxford: Oxford University Press.

- 2008. Our Knowledge of the Internal World. Oxford: Oxford University Press.

Stich, S. e F. Warfield (orgs.) 2003. The Blackwell Guide to the Philosophy of Mind. Oxford: Blackwell.

Stoljar, D. 2005. Physicalism and Phenomenal Concepts. Mind and Language 20: 469-94.

Tye, M. 2003. A Theory of Phenomenal Concepts. Royal Institute of Philosophy Supplement 53: 91-105.

- 2009. Consciousness Revisited. Cambridge, MA: MIT Press.

Wilson MendonçA Universidade Federal do Rio de Janeiro Centro de Ética e Filosofia da Mente Rio de Janeiro BRASIL mendonca@ifcs.ufrj.br Julia Telles Menezes Universidade Federal do Rio de Janeiro

Principia 15(3): 415-438 (2011). 
Centro de Ética e Filosofia da Mente

Rio de Janeiro

BRASIL

juliatelles@gmail.com

Resumo. A ideia intuitivamente plausível de que pelo menos alguns de nossos estados mentais teriam aspectos fenomenais qualitativos aos quais nós teríamos um acesso cognitivo privilegiado é considerada por muitos filósofos como incompatível com a ontologia fisicista. Alguns defensores radicais do fisicismo preferem simplesmente negar a existência de aspectos qualitativos, ao passo que outros materialistas procuram reinterpretar a cognição do caráter fenomenal da nossa experiência do mundo como a aquisição de uma habilidade, isto é, como uma forma de know-how, em oposição à aquisição de informação (know-that). $\mathrm{O}$ presente trabalho expõe e examina criticamente algumas tentativas recentes de compatibilização da natureza sui generis da apreensão cognitiva do caráter fenomenal da nossa experiência com a tese materialista de que o conteúdo proposicional dessa cognição é constituído exclusivamente por fatos físicos.

Palavras-chave: Qualia; conhecimento fenomenal; conceitos fenomenais; contextualismo epistêmico.

Principia 15(3): 415-438 (2011). 